\title{
WSRT 1.4 and 5-GHz light curves for WR 147 (AS 431, WN8(h)+OB)^
}

\author{
D. Y. A. Setia Gunawan ${ }^{1,2}$, A. G. de Bruyn ${ }^{3,1}$, K. A. van der Hucht ${ }^{2}$, and P. M. Williams ${ }^{4}$ \\ 1 Kapteyn Astronomical Institute, PO Box 800, 9700 AV Groningen, The Netherlands \\ 2 Space Research Organization Netherlands, Sorbonnelaan 2, 3584 CA Utrecht, The Netherlands \\ 3 Netherlands Foundation for Research in Astrophysics, PO Box 2, 7990 AA Dwingeloo, The Netherlands \\ ${ }^{4}$ Institute for Astronomy, University of Edinburgh, Royal Observatory, Blackford Hill, Edinburgh EH9 3HJ, UK
}

Received 7 July 2000 / Accepted 12 December 2000

\begin{abstract}
The results of more than 8-yr monitoring (1988-1997) of the Wolf-Rayet binary WR 147 (WN8(h)+OB) with the Westerbork Synthesis Radio Telescope (WSRT) are presented. When the strong winds of the Wolf-Rayet (WR) and OB binary components collide, they produce non-thermal excess radiation in the region where the two winds interact. The binary system, monitored at 1.4 and $5 \mathrm{GHz}(21$ and $6 \mathrm{~cm})$, is not resolved by the WSRT, thus we observed the total flux density of the system. The time-averaged 5 and 1.4-GHz flux densities are $35.4 \pm 0.4 \mathrm{mJy}$ and $26.4 \pm 0.3 \mathrm{mJy}$, respectively. These give a time-averaged spectral index of $\alpha_{5-1.4 \mathrm{GHz}} \approx 0.23 \pm 0.04$, where $S_{\nu} \propto \nu^{\alpha}$. The departure from the value expected for thermal radiation from a spherically symmetric stellar wind, $\alpha=0.6$, can be attributed to non-thermal emission from a bow-shaped source to the north of the thermal source associated with the WN8 star. With a possible detection at $350 \mathrm{MHz}$ of $16 \pm 4 \mathrm{mJy}$, in our separate study of the Cygnus region, the spectral energy distribution, after the contribution of the southern thermal source is subtracted, can be fitted by a synchrotron emission model which includes free-free absorption. The nonthermal emission originates in the region where the winds of the binary components collide. This region, therefore, contains a mixture of relativistic particles accelerated by shocks and thermal particles, responsible for the free-free absorption. We show, in a simplified model of the system, that additional free-free absorption may occur when the line of sight to the collision region passes through the radiophotosphere of the WR wind. The 1.4-GHz flux density of WR 147 varied between $\sim 20$ mJy and $\sim 30 \mathrm{mJy}$. We attribute the irregular, stochastic variations with a typical timescale of about 60 days to inhomogeneities in the wind, with different mechanisms involved in the flux-density increase than in the flux-density decrease. A flux-density increase results when the inhomogeneities in the wind/clumps enter the wind collision region, fuelling the synchrotron emission. The typical timescale of the flux-density decrease is shorter than the timescale of synchrotron loss $\left(\sim 10^{3} \mathrm{yr}\right)$ or the Inverse-Compton lifetime $(\approx 4.5 \mathrm{yr})$, but of the order of the flow time in the colliding-wind region $(\sim 80 \mathrm{~d})$. Therefore, we suggest that the flux-density decrease is due to plasma outflow from the system. Furthermore, variable free-free absorption due to large clumps passing the line of sight may also cause variations in the flux density. We observe a possible long-term flux-density variation on top of the stochastic variation. This variation is fitted with a sinusoid with a $\sim 7.9$-yr period, with a reduced $\chi^{2}$ of 1.9 . However, as the period of the sinusoid is too close to the monitoring time span, further monitoring is needed to confirm this long-term variation.
\end{abstract}

Key words. stars: binaries: general - stars: Wolf-Rayet - stars: individual: WR 147 - radio continuum: stars

\section{Introduction}

The Wolf-Rayet system WR 147 (AS 431, WN8(h)+OB) is, at $d=0.65 \mathrm{kpc}$ (Morris et al. 2000), the second closest WR star after $\gamma^{2}$ Velorum (WR 11, $d=0.26 \mathrm{kpc}$, van der Hucht et al. 1997). It is projected towards the heavily

Send offprint requests to: D. Y. A. Setia Gunawan,

e-mail: D.Y.A.Setia-Gunawan@astro.rug.nl

* Based on observations made with the Westerbork Synthesis Radio Telescope (WSRT), operated by the Netherlands Foundation for Research in Astronomy (NFRA). obscured Cygnus OB2 region and, in spite of its proximity, suffers from substantial optical extinction, with $A_{V}=11.2$ (Morris et al. 2000). WR 147 is among the brightest WR stars at radio frequencies.

The first high-resolution 5-GHz MERLIN observation by Moran et al. (1989) demonstrated that the radio emission originates from two spatially separated components. They showed that the southern, thermal, component coincided with the optical image of the WN8 star; they identified the other component, $00^{\prime \prime} 6$ to the north, as a possible 
source of non-thermal emission. From multi-epoch multifrequency VLA observations, Churchwell et al. (1992) confirmed that the northern component was a non-thermal emitter having a spectral index $\alpha_{2-6 \mathrm{~cm}} \approx-0.5$, where $S_{\nu} \propto \nu^{\alpha}$. The southern component had $\alpha_{2-6 \mathrm{~cm}} \approx+0.6$, consistent with free-free emission from the wind of the WN8 star. At $2 \mathrm{~cm}$, the two components appeared to be joined by a bridge of radio emission, indicating that the sources are spatially related rather than being a chance alignment.

A higher-resolution MERLIN 5-GHz image of WR 147 by Williams et al. (1997) resolved the two components, $\mathrm{N}$ and $\mathrm{S}$, with a separation of $575 \pm 15$ mas. They confirmed that the southern component was associated with the WN8 star, while the northern component was nonthermal, and they associated the latter with the region where the winds of the WN8 star and a possible OB companion collide. Williams et al. (1997) also obtained a MERLIN 1.6-GHz image which showed component $\mathrm{N}$ clearly, but at the position of component S only a $3 \sigma$ signal was apparent. It should be noted that the MERLIN flux densities presented by Williams et al. (1997) are significantly lower than the VLA data by Churchwell et al. (1992) and the WSRT data presented by van der Hucht et al. (1995). They suggested that this may be due to the possibility that a significant fraction of the $5-\mathrm{GHz}$ flux from WR 147 came from a more extended region than observed by MERLIN, which is only capable of detecting emission on scales up to about 700 mas at $5 \mathrm{GHz}$ (Williams et al. 1997).

An UKIRT $K$-band image showed a companion star, with $\Delta K=3.04 \pm 0.09 \mathrm{mag}$ fainter, at a projected angular separation of $635 \pm 20$ mas, with the adopted distance of $0.65 \mathrm{kpc}$ corresponding to a projected linear separation of $413 \pm 13$ AU. The companion has the $K$-luminosity of a B0.5V star (Williams et al. 1997). They also showed that the optical-infrared-radio spectral index of the system is $\alpha=+0.66 \pm 0.02$, appropriate for a stellar wind spectrum.

HST-WFPC2 observations by Niemela et al. (1998) resolved the companions of WR 147 in $U B V$ and, confirming the binarity discovered at infrared wavelengths, showed a projected angular separation of $643 \pm 157$ mas. The corresponding projected linear separation between the WR and OB binary components is $418 \pm 102$ AU. Both stars show similar $B-V$ colours of around $4.1 \mathrm{mag}$, indicating that they are related objects, with the southern WN8 star being two magnitudes brighter than the northern companion. Based on the assumption that the WN8 star has $M_{V}=-6.8$, Niemela et al. (1998) suggested that the northern star should have $M_{V}=-4.7$, and could be an O8-9 V-III or an early B-type star. The spectrum of the companion still needs to be formally classified, which, on account of its faintness relative to that of the WN8 star, will require spatial resolution of the two spectra.

From monitoring of WR 147 at 5 and $1.4 \mathrm{GHz}$ with the WSRT, van der Hucht et al. (1995) found that the combined flux density varied by $25 \%$ at $5 \mathrm{GHz}$ and by $40 \%$ at $1.4 \mathrm{GHz}$ on a timescale of weeks. Contreras \& Rodríguez (1999) also observed a $25 \%$ flux-density increase, notably of the southern source, in their 8.44-GHz VLA images of WR 147, taken in 1995 July and 1996 December.

It is tempting to compare WR 147 with the collidingwind binary WR 146, of which the 1.4-GHz WSRT light curve shows variations on a range of timescales: a steady flux-density increase during 10 years of monitoring, which was also found in the $5-\mathrm{GHz}$ light curve; a 3.4-yr sinusoidal variation; and a rapid, non-periodic variation on a timescale of the order of weeks to months (Setia Gunawan et al. 2000, hereafter Paper I). Variability was also seen in the binary system WR 140 (WC7+O4-5), which shows a clear 7.94-yr period in its IR light curves, optical radialvelocity (RV) curve, and radio light curves (Williams et al. 1990, 1994; White \& Becker 1995), and in its UV RV curve (Setia Gunawan et al. 1995a, 1995b). Models proposed therein discuss the variations in terms of modulation, due to binary motion, of radiation induced by the colliding stellar winds of the two components, and in the case of WR 146, the possibility of a third component. In both WR 140 and WR 146, the observed non-thermal radio emission has been shown to originate in the region where the winds of the WR star and the O-type companion interact. Therefore, we re-investigated the available WSRT data of WR 147, supplemented with more recent data, and reduced them in a more homogeneous manner.

This paper is organized in the following manner. In Sect. 2 the observations and data reduction are explained, while the results are presented in Sect. 3. The discussion, in Sect. 4, is divided into three subsections: Sect. 4.1 presents the spectral energy distribution and the geometry of the system; Sect. 4.2 discusses the origin of the non-thermal emission in WR 147; and Sect. 4.3 discusses the flux-density variability. The conclusions and suggestions for future work are presented in Sect. 5 .

\section{Observations and data reduction}

The WR 147 binary system has been monitored by us with the WSRT at 5 and $1.4 \mathrm{GHz}$ since 1988 . The radio components of WR 147, resolved by MERLIN and the VLA, cannot be resolved by the WSRT and thus we observed the total flux of the system. We find no evidence for emission on scales $\gtrsim 2^{\prime \prime}$. The observations and data reduction of WR 147 were performed in a manner similar to that of WR 146 described in Paper I. For details of the WSRT, the detectors used, the noise and reduction steps, we refer to Paper I. Below we give a summary.

Most of the data were taken in snapshots of a few hours, but occasionally they were obtained in full $12 \mathrm{~h}$ observing runs. The observations employed either the Digital Continuum Backend (DCB) or the Digital Line Backend (DLB). The $5-\mathrm{GHz}$ DCB has a total bandwidth of $80 \mathrm{MHz}$, in 8 adjacent bands of $10 \mathrm{MHz}$, centred at $4874 \mathrm{MHz}$. The 1.4-GHz DCB prior to 1991 had a total bandwidth of $40 \mathrm{MHz}$, centred at $1401 \mathrm{MHz}$ and from 1991 to 1998 had a total bandwidth of $60 \mathrm{MHz}$, centred at $1395 \mathrm{MHz}$. 


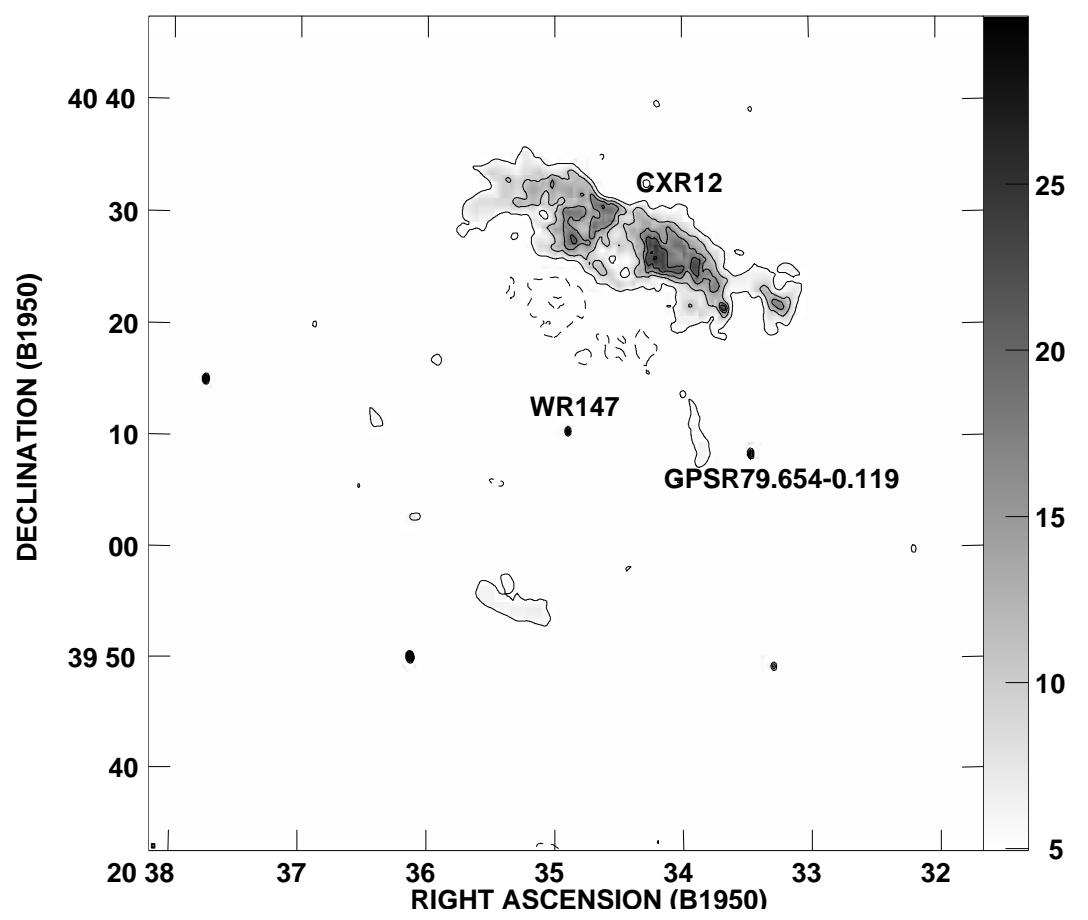

Fig. 1. WSRT 1.4-GHz field around WR 147. Another bright point source in the field, identified with GPSR 79.654-0.119, was used to monitor systematic errors in the data. The map has been corrected for the primary beam. The contour levels represent $-5,5,10,15,20,25,30 \mathrm{mJy}$, where the negative contours are denoted by dashed lines

Of the 1.4-GHz DLB observations we only used data of the continuum channel which has a $10-\mathrm{MHz}$ bandwidth, centred at $1412 \mathrm{MHz}$. These different settings affect the noise of the data. The log of observations is presented in Table 1 for the 1.4-GHz observations and in Table 2 for the $5-\mathrm{GHz}$ observations.

The reduction of the data, which includes calibration and mapping, was carried out with the WSRT NEWSTAR software package (Noordam 1994) in three steps.

i) The data were flux-calibrated. The antenna corrections were obtained from one of the major calibrators: 3C48, $3 \mathrm{C} 147$ or $3 \mathrm{C} 286$, observed just before and/or after the WR 147 observations. The flux densities of these calibrators are on the scale of Baars et al. (1977).

ii) We made maps of the field in both frequencies and obtained 5-GHz and 1.4-GHz models that represent the field. The $0.6 \times 0.65-\mathrm{GHz}$ map of the field around WR 147 was made from a $12 \mathrm{~h}$ synthesis, with a $3^{\prime \prime} .5 \times 5^{\prime \prime}$ beam. In this map, we did not detect any source with a flux density $>0.4 \mathrm{mJy}$, other than WR 147 . Therefore, the $5-\mathrm{GHz}$ model of the field consists of only WR 147. However, at $1.4 \mathrm{GHz}$, the $2^{\circ} \times 2^{\circ}$ map of the field around WR 147 (Fig. 1), made from a $2 \times 12^{\mathrm{h}}$ synthesis, with a $12^{\prime \prime} .5 \times 16^{\prime \prime} 3$ beam, shows other bright point sources and the extended structure of Cygnus-X ridge CXR 12 (Wendker 1991), $15^{\prime}$ north of WR 147, spanning a $\sim 40^{\prime}$ area. Our $1.4-\mathrm{GHz}$ model consists of 22 point sources, down to $2.5 \mathrm{mJy}$, and 5775 components of extended sources with a total flux density of $\sim 7 \mathrm{Jy}$.

iii) The model visibilities were compared with the individual observations using a uv-based modelling program in NEWSTAR. In this program, the fluxes and positions of the point sources in the model are fitted to the observed visibilities using a least-square fit. This fitting procedure is iterated until the values converged. To minimize confusion by extended sources, we did not use data from baselines shorter than $324 \mathrm{~m}$.

The theoretical thermal noise for a $12 \mathrm{~h}$ observation at $1.4 \mathrm{GHz}$ using 5 channels of $10-\mathrm{MHz}$ bandwidth, 40 baselines and two polarizations is about $0.06 \mathrm{mJy}$, and $0.15 \mathrm{mJy}$ for a typical $2 \mathrm{~h}$ observation. However, sidelobe confusion by the extended source CXR 12 adds extra noise and we consider $0.25 \mathrm{mJy}$ to be typical for a $2 \mathrm{~h}$ observation. The theoretical thermal noise at $5 \mathrm{GHz}$ of the $80-\mathrm{MHz}$ bandwidth ( 8 bands of $10 \mathrm{MHz}$ bandwidth, 40 baselines, two polarizations) with $12 \mathrm{~h}$ observing time, is $0.07 \mathrm{mJy}$, and about $0.17 \mathrm{mJy}$ for a $2 \mathrm{~h}$ observation. The use of different calibrators introduced extra uncertainty as well, which we adopt to be $1 \%$ of the flux density. The errors in the flux densities listed in Tables 1 and 2 include all three contributions: thermal noise, uncertainty due to different calibrators, and, at $1.4 \mathrm{GHz}$, side-lobe confusion. A detailed discussion on the sources of error is given in Paper I.

\section{Results}

The results of the observations are presented in Figs. 2 and 8 . The calibrators for the $1.4-\mathrm{GHz}$ data taken in 1992.361 were observed 4 days after the observation of WR 147, possibly with very different antenna and weather conditions, which makes the accuracy of this measurement uncertain. This data point was not used in further analysis. 
Table 1. Log of the WSRT 1.4-GHz observations

\begin{tabular}{|c|c|c|c|c|c|c|c|}
\hline year & $\begin{array}{l}\text { I.T. } \\
\text { hh:mm }\end{array}$ & $\begin{array}{c}S_{1.4 \mathrm{GHz}} \\
(\mathrm{mJy})\end{array}$ & calibrator & year & $\begin{array}{l}\text { I.T. } \\
\text { hh:mm }\end{array}$ & $\begin{array}{c}S_{1.4 \mathrm{GHz}} \\
(\mathrm{mJy})\end{array}$ & calibrator \\
\hline 1989.027 & $05: 35$ & $25.84 \pm 0.46$ & $3 \mathrm{C} 147$ & 1994.668 & $04: 53$ & $25.23 \pm 0.49$ & $3 \mathrm{C} 147$ \\
\hline 1989.794 & 01:29 & $28.13 \pm 0.58$ & $3 \mathrm{C} 286$ & 1994.676 & $03: 25$ & $25.61 \pm 0.56$ & $3 \mathrm{C} 147$ \\
\hline 1989.808 & 02:02 & $26.86 \pm 0.57$ & $3 \mathrm{C} 48$ & 1994.687 & $01: 50$ & $25.24 \pm 0.68$ & $3 \mathrm{C} 286$ \\
\hline 1989.898 & $01: 55$ & $27.64 \pm 1.19$ & $3 \mathrm{C} 147$ & 1994.695 & $05: 31$ & $24.97 \pm 0.54$ & $3 \mathrm{C} 147$ \\
\hline 1989.917 & $01: 28$ & $27.77 \pm 0.62$ & $3 \mathrm{C} 147$ & 1994.720 & 05:09 & $25.63 \pm 0.50$ & $3 \mathrm{C} 48$ \\
\hline 1989.939 & $02: 30$ & $28.68 \pm 1.06$ & $3 \mathrm{C} 147$ & 1994.731 & $03: 10$ & $25.29 \pm 0.58$ & $3 \mathrm{C} 48$ \\
\hline 1989.961 & $01: 55$ & $28.75 \pm 1.15$ & $3 \mathrm{C} 147$ & 1994.742 & $02: 55$ & $25.20 \pm 0.56$ & $3 \mathrm{C} 48$ \\
\hline 1989.975 & $12: 00$ & $39.26 \pm 0.48$ & $3 \mathrm{C} 147$ & 1994.958 & $03: 51$ & $25.94 \pm 0.51$ & $3 \mathrm{C} 48$ \\
\hline 1990.008 & $06: 55$ & $27.70 \pm 0.50$ & $3 \mathrm{C} 147$ & 1994.966 & $05: 47$ & $26.48 \pm 0.48$ & $3 \mathrm{C} 147$ \\
\hline 1990.033 & $02: 26$ & $28.20 \pm 1.05$ & $3 \mathrm{C} 48$ & 1994.980 & 05:49 & $26.30 \pm 0.47$ & $3 \mathrm{C} 147$ \\
\hline 1990.057 & 01:12 & $27.77 \pm 0.68$ & $3 \mathrm{C} 147$ & 1995.030 & $04: 28$ & $26.24 \pm 0.49$ & $3 \mathrm{C} 147$ \\
\hline 1990.501 & $01: 55$ & $28.22 \pm 0.64$ & $3 \mathrm{C} 147$ & 1995.452 & $05: 22$ & $24.94 \pm 0.47$ & $3 \mathrm{C} 48$ \\
\hline 1990.539 & $02: 55$ & $25.54 \pm 0.50$ & $3 \mathrm{C} 147$ & 1995.463 & $00: 59$ & $25.28 \pm 0.83$ & $3 \mathrm{C} 147$ \\
\hline 1990.559 & $08: 33$ & $27.54 \pm 0.46$ & $3 \mathrm{C} 147$ & 1995.468 & $00: 59$ & $24.91 \pm 0.89$ & $3 \mathrm{C} 48$ \\
\hline 1990.586 & $05: 59$ & $26.41 \pm 0.46$ & $3 \mathrm{C} 147$ & 1995.474 & $00: 59$ & $24.89 \pm 0.80$ & $3 \mathrm{C} 48$ \\
\hline 1990.632 & 07:19 & $26.57 \pm 0.45$ & $3 \mathrm{C} 147$ & 1995.485 & $00: 59$ & $25.05 \pm 0.83$ & $3 \mathrm{C} 48$ \\
\hline 1990.654 & 03:11 & $24.89 \pm 0.89$ & $3 \mathrm{C} 286$ & 1995.496 & $00: 58$ & $24.73 \pm 0.79$ & $3 \mathrm{C} 48$ \\
\hline 1990.684 & $05: 56$ & $27.73 \pm 0.49$ & $3 \mathrm{C} 147$ & 1995.504 & $00: 58$ & $24.29 \pm 0.73$ & $3 \mathrm{C} 48$ \\
\hline 1992.312 & $00: 59$ & $27.51 \pm 0.78$ & 3C147 & 1995.512 & 01:02 & $24.67 \pm 0.75$ & $3 \mathrm{C} 48$ \\
\hline 1992.361 & $01: 59$ & $29.47 \pm 0.64$ & $3 \mathrm{C} 286$ & 1995.520 & $00: 59$ & $24.57 \pm 0.80$ & $3 \mathrm{C} 48$ \\
\hline 1992.413 & $01: 57$ & $26.72 \pm 0.64$ & $3 \mathrm{C} 48$ & 1995.531 & $00: 59$ & $25.46 \pm 0.78$ & $3 \mathrm{C} 48$ \\
\hline 1992.534 & $01: 59$ & $27.24 \pm 0.62$ & $3 \mathrm{C} 147$ & 1995.589 & $05: 30$ & $24.76 \pm 0.46$ & $3 \mathrm{C} 48$ \\
\hline 1992.550 & $05: 44$ & $27.29 \pm 0.59$ & $3 \mathrm{C} 147$ & 1995.600 & $02: 53$ & $24.62 \pm 0.51$ & $3 \mathrm{C} 48$ \\
\hline 1992.613 & $01: 58$ & $27.03 \pm 0.60$ & $3 \mathrm{C} 147$ & 1995.602 & $02: 52$ & $24.66 \pm 0.53$ & $3 \mathrm{C} 48$ \\
\hline 1992.646 & $01: 57$ & $26.11 \pm 0.63$ & $3 \mathrm{C} 48$ & 1995.616 & $07: 21$ & $24.86 \pm 0.46$ & $3 \mathrm{C} 48$ \\
\hline 1993.542 & $01: 59$ & $24.77 \pm 0.62$ & $3 \mathrm{C} 147$ & 1995.635 & $05: 47$ & $24.83 \pm 0.46$ & $3 \mathrm{C} 48$ \\
\hline 1993.550 & $02: 23$ & $25.37 \pm 0.55$ & $3 \mathrm{C} 286$ & 1995.649 & $05: 52$ & $25.15 \pm 0.47$ & $3 \mathrm{C} 147$ \\
\hline 1993.553 & $02: 27$ & $25.27 \pm 0.55$ & $3 \mathrm{C} 147$ & 1995.671 & $01: 55$ & $24.61 \pm 0.66$ & $3 \mathrm{C} 147$ \\
\hline 1993.564 & $04: 25$ & $25.27 \pm 0.52$ & $3 \mathrm{C} 147$ & 1995.698 & $03: 34$ & $24.99 \pm 0.51$ & $3 \mathrm{C} 48$ \\
\hline 1993.613 & $01: 55$ & $24.56 \pm 0.57$ & $3 \mathrm{C} 147 / 3 \mathrm{C} 286$ & 1996.386 & $05: 58$ & $25.13 \pm 0.47$ & $3 \mathrm{C} 48$ \\
\hline 1993.638 & $01: 58$ & $25.33 \pm 0.66$ & $3 \mathrm{C} 147$ & 1996.422 & $04: 58$ & $25.34 \pm 0.51$ & $3 \mathrm{C} 147$ \\
\hline 1993.663 & $01: 59$ & $24.62 \pm 0.57$ & $3 \mathrm{C} 286$ & 1996.493 & $05: 27$ & $25.79 \pm 0.50$ & $3 \mathrm{C} 147$ \\
\hline 1993.709 & 02:00 & $25.31 \pm 0.70$ & $3 \mathrm{C} 147$ & 1996.559 & $01: 27$ & $24.75 \pm 0.83$ & $3 \mathrm{C} 48$ \\
\hline 1993.758 & $02: 47$ & $25.31 \pm 0.54$ & $3 \mathrm{C} 147$ & 1997.367 & $03: 59$ & $27.77 \pm 0.52$ & $3 \mathrm{C} 48$ \\
\hline 1993.903 & 03:01 & $25.31 \pm 0.96$ & $3 \mathrm{C} 147$ & 1997.389 & 03:05 & $27.37 \pm 0.65$ & $3 \mathrm{C} 48$ \\
\hline 1993.936 & 03:00 & $24.70 \pm 0.95$ & $3 \mathrm{C} 48$ & 1997.424 & $11: 00$ & $27.94 \pm 0.58$ & $3 \mathrm{C} 48$ \\
\hline 1993.975 & $02: 23$ & $24.33 \pm 0.55$ & $3 \mathrm{C} 48$ & 1997.449 & $09: 23$ & $27.33 \pm 0.48$ & $3 \mathrm{C} 147$ \\
\hline 1994.016 & $02: 17$ & $24.82 \pm 0.57$ & $3 \mathrm{C} 147$ & 1997.485 & $03: 54$ & $27.29 \pm 0.57$ & $3 \mathrm{C} 286$ \\
\hline 1994.088 & 01:48 & $24.77 \pm 0.61$ & $3 \mathrm{C} 147$ & 1997.496 & $05: 10$ & $27.73 \pm 0.56$ & $3 \mathrm{C} 147$ \\
\hline 1994.104 & 03:07 & $23.79 \pm 0.51$ & $3 \mathrm{C} 48$ & 1997.504 & 09:24 & $26.66 \pm 0.48$ & $3 \mathrm{C} 48$ \\
\hline 1994.120 & $02: 59$ & $23.93 \pm 0.52$ & $3 \mathrm{C} 48$ & 1997.528 & $04: 56$ & $27.91 \pm 0.57$ & $3 \mathrm{C} 48$ \\
\hline 1994.181 & $03: 51$ & $23.79 \pm 0.49$ & $3 \mathrm{C} 48$ & 1997.632 & $05: 57$ & $27.12 \pm 0.50$ & $3 \mathrm{C} 286$ \\
\hline 1994.641 & $01: 57$ & $25.25 \pm 0.63$ & $3 \mathrm{C} 48$ & 1997.687 & $04: 59$ & $27.09 \pm 0.52$ & $3 \mathrm{C} 147$ \\
\hline 1994.649 & $10: 00$ & $25.36 \pm 0.44$ & $3 \mathrm{C} 147$ & & & & \\
\hline
\end{tabular}

Table 2. Log of the WSRT 5-GHz observations

\begin{tabular}{llcl|llll}
\hline year & $\begin{array}{l}\text { I.T. } \\
\text { hh:mm }\end{array}$ & $\begin{array}{c}S_{5 \mathrm{GHz}} \\
(\mathrm{mJy})\end{array}$ & calibrator & year & $\begin{array}{l}\text { I.T. } \\
\text { hh:mm }\end{array}$ & $\begin{array}{l}S_{5 \mathrm{GHz}} \\
(\mathrm{mJy})\end{array}$ & calibrator \\
\hline 1988.471 & $01: 59$ & $35.85 \pm 0.52$ & 3C286 & 1990.353 & $03: 50$ & $35.15 \pm 0.39$ & 3C286 \\
1988.501 & $02: 24$ & $33.87 \pm 0.42$ & 3C286 & 1990.370 & $00: 59$ & $36.67 \pm 0.50$ & 3C48 \\
1989.564 & $01: 44$ & $34.01 \pm 0.47$ & 3C286 & 1990.400 & $01: 24$ & $36.75 \pm 0.47$ & 3C286 \\
1989.608 & $00: 59$ & $35.41 \pm 0.52$ & 3C147 & 1990.454 & $02: 58$ & $35.41 \pm 0.40$ & 3C286 \\
1989.616 & $02: 09$ & $35.66 \pm 0.44$ & 3C286 & 1993.107 & $03: 25$ & $35.42 \pm 0.42$ & 3C286 \\
1989.646 & $01: 57$ & $36.96 \pm 0.45$ & 3C286 & 1993.129 & $01: 59$ & $36.95 \pm 0.47$ & 3C48 \\
1989.712 & $00: 59$ & $35.45 \pm 0.53$ & 3C147 & 1993.471 & $03: 43$ & $35.95 \pm 0.40$ & 3C147 \\
1989.764 & $03: 00$ & $36.67 \pm 0.43$ & 3C286 & 1995.422 & $03: 54$ & $36.91 \pm 0.42$ & 3C 48 \\
& & & 1997.162 & $04: 23$ & $27.00 \pm 0.51$ & 3C286 \\
\hline
\end{tabular}




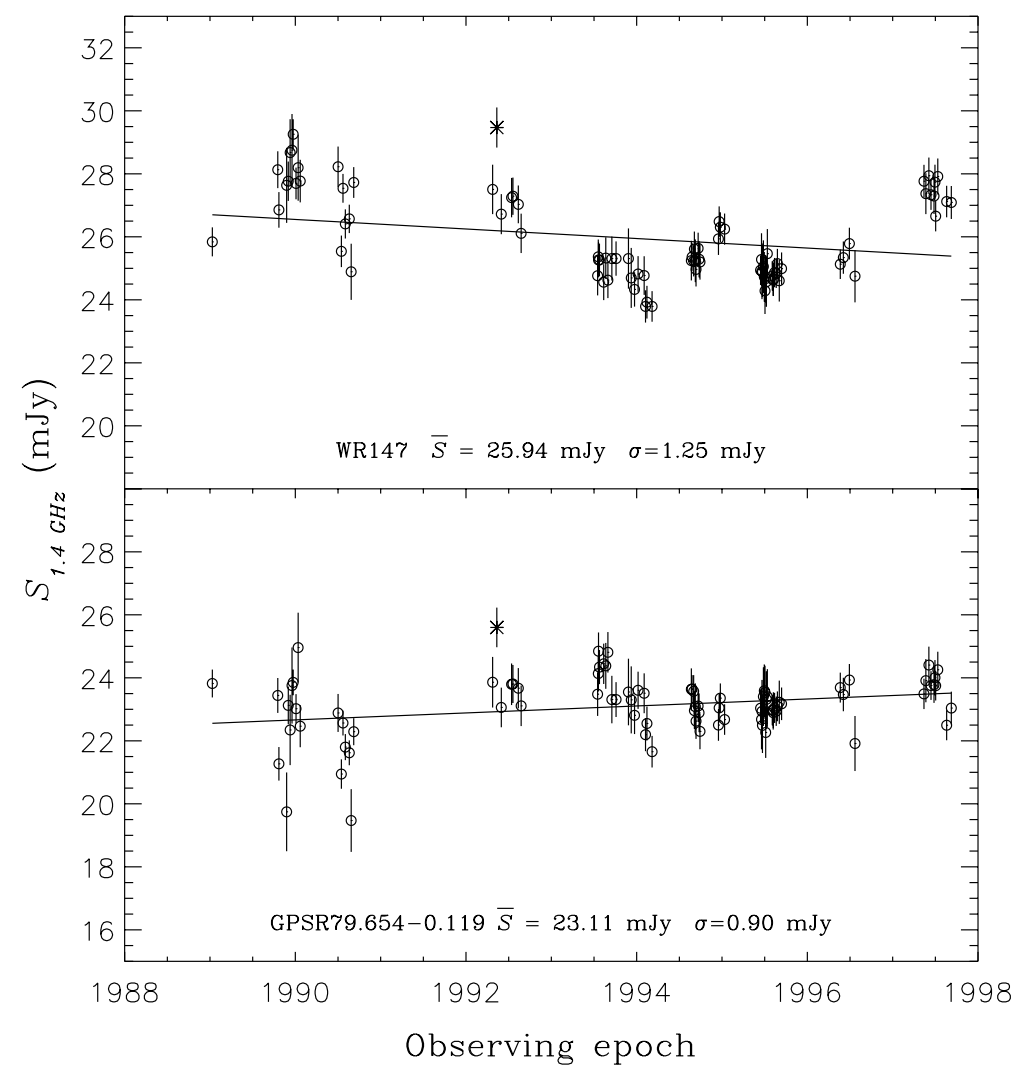

Fig. 2. The 1.4-GHz light curves of WR 147 and GPSR 79.654-0.119. For the purpose of comparison, we show here the "apparent" flux density, i.e., not corrected for primary beam. The data point marked with $(*)$ is not used for further analysis

As in Paper I, we have tried to find sources in the field which can be used as control sources to check for systematic variations in the data due to instrumental or atmospheric effects. There is only one point source in the 1.4-GHz field which can be used as a control source, although it is located about $990^{\prime \prime}$ away from the field centre. With the aid of SIMBAD, we identified this source with the object GPSR 79.654-0.119, from the Galactic Plane Survey of Zoonematkermani et al. (1990), the object 19P42 in the list of Wendker et al. (1991), and the object WSRTGP 2033+4008 in the list of Taylor et al. (1996). In Table 3 we list the flux densities and positions of WR 147 and GPSR 79.6540.119/19P42/ WSRTGP $2033+4008$, where the 5 -GHz position and flux density of GPSR 79.654-0.119 are adopted from Zoonematkermani et al. (1990). The 1.4-GHz flux density of 19P42, from observation in 1985 July/August, is in accordance with our time-averaged flux density. This suggests that at $1.4 \mathrm{GHz}$ the object did not vary in the 10-year interval. The $327-\mathrm{MHz}$ WSRT Galactic Plane Survey of Taylor et al. (1996), done in 1984-1985, gave a higher flux density of $80 \pm 3$ mJy than the $350-\mathrm{MHz}$ flux density found by $59 \pm 3$ mJy of Setia Gunawan et al. (in prep., hereafter Paper II) in an observation done in 1995. This suggests a flux density variation at $350-\mathrm{MHz}$ in the 10 -year interval. The 350 to $5000-\mathrm{MHz}$ spectral indices of GPSR 79.654-0.119 in Table 3 suggest a continuous fluxdensity increase in the low-frequency region and that no absorption is present at low frequency.
We obtain linear fits, by using a least-square method, to the data of WR 147 and GPSR 79.654-0.119, and compare the differences in flux density with these fits in both objects. Dividing the flux-density differences by the corresponding fits, we look for correlation between the flux-density variations in the two objects. As shown in Fig. 3, the correlation coefficient is quite low $(=0.22)$, suggesting that the data are not correlated. Hence, it is not necessary to correct the flux densities of WR 147 using the variations in GPSR 79.654-0.119. The linear fit shows a slow trend over the 8.66-year monitoring period where the average flux density of WR 147 changed from $26.7 \mathrm{mJy}$ to $25.4 \mathrm{mJy}$, with a mean of $25.9 \mathrm{mJy}$, i.e., by $-0.6 \% / y r$. This value is within the noise of the observations, and, therefore, we will not consider this slow trend in the analysis of the data.

We note that at a distance of $990^{\prime \prime}$, the primary-beam attenuation of GPSR 79.654-0.119 is about 1.78. Pointing errors of $20^{\prime \prime}$ would then cause an apparent flux-density error of $1.7 \%$. We attribute the outliers in Fig. 3 to this small pointing error. Despite its sensitivity to pointing error, GPSR 79.654-0.119 shows a small flux-density variability ( $\sim 3 \%)$, which is about the noise of the data, while WR 147 shows a wider spread $(\sim 10 \%)$. We conclude that the 1.4-GHz flux-density variability in WR 147 is real and not due to observational error.

At $5 \mathrm{GHz}$, we cannot find any object suitable as control source. Therefore, we cannot check whether the low flux density in the 1997 data is due to systematic instrumental 
Table 3. Flux densities and positions of WR 147 and GPSR 79.654-0.119. The flux densities were obtained from our 1.4 and 5-GHz maps of the field. The 350-MHz flux density is from the mosaic observation of Setia Gunawan et al. (in prep., Paper II). All flux densities have been corrected for the primary beam

\begin{tabular}{l|cc|cc}
\hline & WR 147 & ref. & GPSR 79.654-0.119 & ref. \\
\hline $\mathrm{S}_{5 \mathrm{GHz}}(\mathrm{mJy})$ & $35.4 \pm 0.4$ & $\mathrm{Se00}$ & 30 & Zo90 \\
$\mathrm{S}_{1.4 \mathrm{GHz}}(\mathrm{mJy})$ & $26.4 \pm 0.3$ & $\mathrm{Se00}$ & $42.9 \pm 0.5$ & $\mathrm{Se00}$ \\
& & & $45 \pm 2$ & We91 \\
$\mathrm{S}_{350 \mathrm{MHz}}(\mathrm{mJy})$ & $16 \pm 4$ & $\mathrm{Se00b}$ & $59 \pm 3$ & $\mathrm{Se00b}$ \\
& & & $80 \pm 3$ & $\mathrm{Ta} 96$ \\
$\alpha_{5-1.4 \mathrm{GHz}}$ & $0.23 \pm 0.04$ & $\mathrm{Se00}$ & $-0.28 \pm 0.04$ & $\mathrm{Se00}$ \\
$\alpha_{1400-350 \mathrm{GHz}}$ & $0.4 \pm 0.2$ & $\mathrm{Se00}$ & $-0.23 \pm 0.08$ & $\mathrm{Se00}$ \\
\hline
\end{tabular}

\begin{tabular}{l|ccc|ccc|c}
\hline & \multicolumn{3}{|c|}{$\mathrm{RA}(1950.0)$} & \multicolumn{3}{|c|}{ DEC (1950.0) } & ref. \\
\hline 5 GHz & $\mathrm{h}$ & $\mathrm{m}$ & $\mathrm{s}$ & & ${ }^{\prime}$ & ${ }^{\prime \prime}$ & \\
WR 147 & & & & & & & \\
GPSR 79.654-0.119 & 20 & 33 & 27.98 & +40 & 08 & 32.7 & Zo90 \\
\hline 1.4 GHz & & & & & & & \\
WR 147 & 20 & 34 & $53.85 \pm 0.03$ & +40 & 10 & $38.7 \pm 0.5$ & Se00 \\
GPSR 79.654-0.119 & 20 & 33 & $27.98 \pm 0.03$ & +40 & 08 & $33.7 \pm 0.5$ & Se00 \\
19P42 & 20 & 33 & $28.2 \pm 0.3$ & +40 & 08 & $30 \pm 5$ & We91 \\
\hline 350 MHz & & & & & & & \\
WR 147 & 20 & 34 & $53 \pm 1$ & +40 & 10 & $39 \pm 12$ & Se00b \\
GPSR 79.654-0.119 & 20 & 33 & $28.0 \pm 0.12$ & +40 & 08 & $36 \pm 2$ & Se00b \\
WSRTGP 2033+4008 & 20 & 33 & $28.10 \pm 0.35$ & +40 & 08 & $33 \pm 5$ & Ta96 \\
\hline
\end{tabular}

References:

Se00: This work; Se00b: Paper II; Ta96: Taylor et al. 1996; We91: Wendker et al. 1991; Zo90: Zoonematkermani et al. 1990.

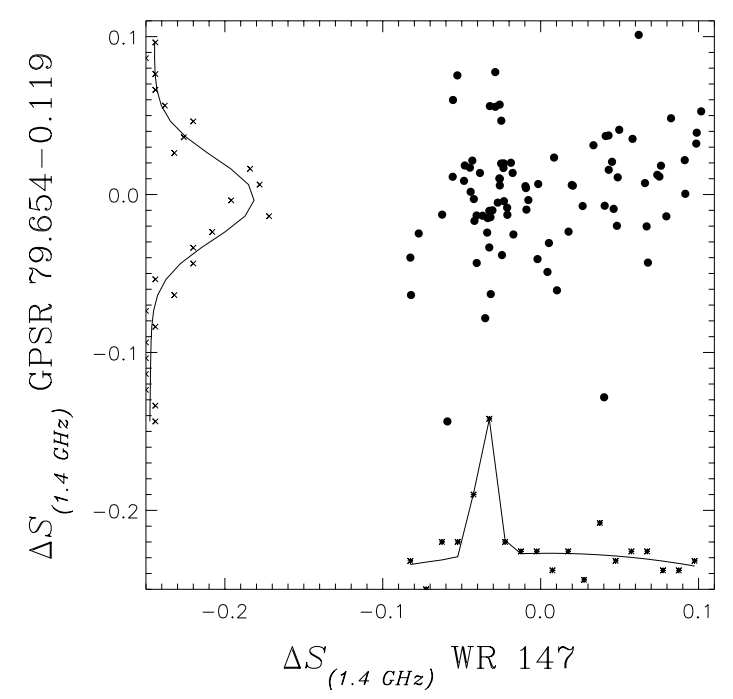

Fig. 3. Correlation between the ratio $\frac{\Delta S}{S_{\text {fit }}}$ for WR 147 and GPSR 79.654-0.119 at $1400 \mathrm{MHz}$. At the bottom and left sides of the figure are histograms for WR 147 and GPSR 79.6540.119 with their Gaussian fits. GPSR 79.654-0.119 follows a Gaussian fit, with $\sigma \approx 3 \%$. However, the histogram of WR 147 has a broad base and cannot be fitted with a Gaussian

error, or atmospheric causes, or a real variation. A linearleast-squares fit of the data of the period of 1988-1995, i.e., excluding the 1997 data, shows that WR 147 has a constant (average) flux density.

\section{Discussion}

The resolved images of WR 147 at 1.6 and $5 \mathrm{GHz}$ by MERLIN (Williams et al. 1997) and at $8.3 \mathrm{GHz}$ by the VLA (Contreras \& Rodríguez 1999) show a southern source with a spectral index typical for a thermal source and a bow-shaped emission to the north of it with a nonthermal spectral index (see Table 5). Combined with the UKIRT near-infrared and HST $U B V$ images, where a star further north of the non-thermal emission can be seen, we see a clear picture of a binary system with a colliding wind region, as modelled by Usov (1992) and Eichler \& Usov (1993). In this model, the non-thermal radiation observed in colliding wind binaries is thought to be synchrotron radiation arising from electrons accelerated to relativistic velocities by first-order Fermi acceleration in shocks in the region where the winds of two binary components collide. A second mechanism, acceleration of electrons to relativistic velocities by magnetic-field compression in colliding winds, has been proposed by Jardine et al. (1996), but still needs further development.

By assuming that equipartition of energy (e.g., Miley 1991) does take place in the synchrotron-production region, the magnetic-field strength at the shock front of 
WR 147, with a size of 267 mas, is $B_{\text {shock }} \approx 5 \mathrm{mG}$. We assume that at large distances, $>3 R_{\star}$, the magnetic field of the star varies inversely with distance (e.g., Eichler \& Usov 1993) according to:

$B \approx B_{\text {surface }} \times \frac{v_{\text {rotation }}}{v_{\infty}} \frac{R_{\star}^{2}}{r_{\mathrm{A}} r}$,

where $R_{\star}$ is the stellar radius, $r_{\mathrm{A}}$ is the Alfven radius, $\sim 1-3 R_{\star}$, and $r$ is the distance from the star. The stellar rotational velocity $v_{\text {rotation }}$ is not well known for WR stars. Taking $r_{\mathrm{A}}=3 R_{\star}$, the surface magnetic field of the binary components is about a few $10^{4} B_{\text {shock }}$, i.e., of the order of a few 10-100 G, depending on the velocity ratio $v_{\text {rotation }} / v_{\infty}$. This is consistent with the model result of Maheswaran \& Cassinelli (1994), who expect that a star with an initial main-sequence mass of $M_{\mathrm{ZAMS}}=60 \mathrm{M}_{\odot}$ will have a surface magnetic field up to a few $100 \mathrm{G}$ during early stages of the WR phase and up to a few $1000 \mathrm{G}$ at the terminal WR stage. The magnetic-field strength derived above is also consistent with the weak field approximation calculated by Ignace et al. (1998) for a WN5 star, where the stellar surface magnetic field needs to be $\ll 30000 \mathrm{G}$.

\subsection{Spectral energy distribution and geometry of the system}

The expected radio and infrared flux densities, $S_{\nu}$, caused by free-free radiation of an early-type star with a smooth, continuous, spherical wind, as derived by Wright \& Barlow (1975), is frequency-dependent according to:

$S_{\nu}=23.2\left(\frac{\dot{M}}{\mu v_{\infty}}\right)^{4 / 3} \frac{\nu^{2 / 3}}{d^{2}} \gamma^{2 / 3} g_{\mathrm{ff}}^{2 / 3} Z^{4 / 3} \mathrm{Jy}$

where $\dot{M}$ is the mass-loss rate in $M_{\odot} \mathrm{yr}^{-1}, v_{\infty}$ is the terminal velocity of the wind in $\mathrm{km} \mathrm{s}^{-1}, d$ is the distance to the star in kpc, $\mu$ is the mean molecular weight, $\gamma$ is the mean number of electrons per ion, $Z$ is the rms ionic charge, and the frequency $\nu$ is in $\mathrm{Hz}$. The Gaunt factor $g_{\mathrm{ff}}$ can be approximated by (Leitherer \& Robert 1991):

$g_{\mathrm{ff}}\left(\nu, T_{\mathrm{w}}\right)=9.77\left[1+0.13 \log \left(\frac{T_{\mathrm{w}}^{3 / 2}}{Z \nu}\right)\right]$,

where $T_{\mathrm{w}}$ is the wind electron temperature. This implies that $\mathrm{S}_{\nu} \propto \nu^{0.6}$.

Williams et al. (1997) derived a spectral index of $\alpha=+0.66 \pm 0.02$ for the thermal source of WR 147 , based on a least-square fit of mid-infrared to radio fluxes. From a near-simultaneous observation, Skinner et al. (1999) obtained $\alpha_{14.94-22.46 \mathrm{GHz}}=+0.62 \pm 0.1$.

In Fig. 4, we have drawn the spectral energy distribution of WR 147, from the flux densities listed in Table 5. We apply a least-square fit to the thermal flux densities, to obtain $\alpha_{\text {thermal }}=+0.60$. This value still includes any fluxdensity variability which may be present in the WR 147 system. Subtracting the linear fit values from the total flux densities, we obtain the "calculated" non-thermal flux density.

Churchwell et al. (1992) determined the mass-loss rate and the mean electron temperature of the wind of the WN8 star by fitting a power-law density distribution to the 15 and $22-\mathrm{GHz}$ VLA visibility data. Assuming $d=630 \mathrm{pc}, v_{\infty}=900 \mathrm{kms}^{-1}, \mu=2.70, Z=1$, and $\gamma=1$, they derived a mass-loss rate of $\dot{M}(\mathrm{WR})=$ $4.2 \pm 0.210^{-5} M_{\odot} \mathrm{yr}^{-1}$ and a wind electron temperature of $T_{\mathrm{w}}=9400 \pm 1000 \mathrm{~K}$. At a distance $d=650 \mathrm{pc}$ and with wind velocity $v_{\infty}(\mathrm{WR})=950 \mathrm{~km} \mathrm{~s}^{-1}, Z_{\mathrm{WR}}=1.005$, $\gamma_{\mathrm{WR}}=1.003, \mu_{\mathrm{WR}}=3.18$, as in Morris et al. (2000), the mass-loss rate is $\dot{M}(\mathrm{WR})=6.5 \pm 0.210^{-5} M_{\odot} \mathrm{yr}^{-1}$.

By comparing ISO-SWS and ground-based optical and infrared data with a non-LTE model that incorporates line-blanketing and clumping in the wind, with filling factors in the range of $0.25-0.04$, and adopting the wind electron temperature of Churchwell et al. (1992), Morris et al. (2000) derived significantly lower values of $\dot{M}(\mathrm{WR})=$ (1.5-3.7) $10^{-5} M_{\odot} \mathrm{yr}^{-1}$. They concluded that a filling factor of 0.1 gave good agreement between the infrared observed and synthetic emission-line profiles and led to a mass-loss rate of $\dot{M}(\mathrm{WR})=2.410^{-5} M_{\odot} \mathrm{yr}^{-1}$, which we will adopt in this paper.

Usov (1992) has modelled colliding winds in massive binaries, i.e., for smooth winds. In this model one can deduce the location where the winds from the binary components collide, which is where the ram-pressures of the winds are equal:

$r_{\mathrm{WR}}=\frac{1}{1+\eta^{\frac{1}{2}}} D_{\mathrm{WR}-\mathrm{OB}}, \quad r_{\mathrm{OB}}=\frac{\eta^{\frac{1}{2}}}{1+\eta^{\frac{1}{2}}} D_{\mathrm{WR}-\mathrm{OB}}$

with $\eta=\frac{\dot{M} v_{\infty}(\mathrm{B})}{\dot{M} v_{\infty}(\mathrm{WR})}$,

where $r_{\mathrm{WR}}$ and $r_{\mathrm{OB}}$ are the distances of the collidingwind region from the WR and OB stars, respectively, and $D_{\mathrm{WR}-\mathrm{OB}}$ is the binary separation.

From the projected binary separation and distance between the WN8 star and the non-thermal source (Williams et al. 1997), we derive the wind-momentum ratio, $\eta=$ 0.011 using Eq. (5). From the adopted mass-loss rate and terminal wind velocity of the WR star and assuming a wind velocity of the $\mathrm{OB}$ star to be $800 \mathrm{kms}^{-1}$, we derive a mass-loss rate for the OB star of $\dot{M}(\mathrm{OB})=$ $410^{-7} M_{\odot} \mathrm{yr}^{-1}$.

The extent of the radiophotosphere of a smooth wind can be expressed as (Wright \& Barlow 1975):

$$
\begin{aligned}
R_{\nu}= & 2.810^{28} Z^{2} g_{\mathrm{ff}}\left(\nu, T_{\mathrm{w}}\right)^{1 / 3} T_{\mathrm{w}}^{-1 / 2} \gamma^{1 / 3} \\
& \times\left(\frac{\dot{M}}{\mu v_{\infty} \nu}\right)^{2 / 3} \mathrm{~cm} .
\end{aligned}
$$

Applying the mass-loss rates and wind velocities and adopting for the WN8 wind similar conditions as in Morris et al. (2000), and for the OB wind typical values of $Z_{\mathrm{B}}=1$, $\gamma_{\mathrm{B}}=1, \mu_{\mathrm{B}}=1.5$, we obtain radii of the radiophotospheres 
Table 4. Measured, derived and estimated parameters of WR 147

\begin{tabular}{|c|c|c|c|}
\hline \multicolumn{4}{|l|}{ System } \\
\hline & & unit & ref. \\
\hline distance to the system & $650_{-110}^{+130}$ & $\mathrm{pc}$ & $\mathrm{Mo00}$ \\
\hline$V$ & 13.72 & mag & Mo00 \\
\hline$M_{V}$ & -6.4 & mag & $\mathrm{Mo00}$ \\
\hline$A_{V}$ & 11.2 & mag & $\mathrm{Mo00}$ \\
\hline$E_{B-V}$ & $4.1 \pm 0.2$ & mag & $\mathrm{Mo} 00$ \\
\hline$R_{V}$ & $2.7 \pm 0.1$ & mag & $\mathrm{Mo00}$ \\
\hline binary separation & $643 \pm 157$ & mas & Ni98 \\
\hline at $650 \mathrm{pc}, i=0^{\circ}$ & 417 & $\mathrm{AU}$ & d \\
\hline binary period $\left(e=0, i=0^{\circ}\right)$ & 1350 & $\mathrm{yr}$ & $\mathrm{d}$ \\
\hline$L_{\mathrm{NT}}(1.4-15 \mathrm{GHz})$ & $1.310^{29}$ & $\operatorname{erg~sec} \sec ^{-1}$ & $\mathrm{~d}$ \\
\hline$B_{\min \mathrm{NT}}$ & 5 & mGauss & $\mathrm{d}$ \\
\hline$L_{\mathrm{X}}($ EINSTEIN-IPC $0.2-4.0 \mathrm{keV})$ & $0.14 \pm 0.02$ & $\left(L_{\odot}\right)$ & Po87 \\
\hline$L_{\mathrm{X}}($ ROSAT-PSPC $\quad 0.2-2.4 \mathrm{keV})$ & $0.02 \pm 0.01$ & $\left(L_{\odot}\right)$ & Po95 \\
\hline
\end{tabular}

\section{Binary components}

\begin{tabular}{|c|c|c|c|c|c|}
\hline & $\overline{\mathrm{WR}}$ & ref. & $\mathrm{OB}$ & ref. & unit \\
\hline $\begin{array}{l}\text { separation T to NT source } \\
\text { at } 650 \mathrm{pc}, i=0^{\circ}\end{array}$ & $\begin{array}{c}575 \pm 15 \\
374\end{array}$ & Wi97 & $\begin{array}{l}68 \\
43\end{array}$ & $\mathrm{~d}$ & $\begin{array}{l}\mathrm{mas} \\
\mathrm{AU}\end{array}$ \\
\hline$V$ & 13.86 & Ni98 & 16.02 & $\mathrm{Ni98}$ & mag \\
\hline$B-V$ & $4.1 \pm 0.1$ & $\mathrm{Ni98}$ & $4.1 \pm 0.3$ & Ni98 & mag \\
\hline $\log L / L_{\odot}$ & 5.52 & Cr95 & $4.7 \pm 0.1$ & Mo00 & \\
\hline spectral type & WN8(h) & Sm96 & $\mathrm{B} 0.5 \mathrm{~V}$ & Wi97 & \\
\hline$M_{V}$ & -6.3 & Mo00 & -4.1 & $\mathrm{Mo00}$ & mag \\
\hline $\log (g)$ & & & 4 & $\mathrm{Mo} 00$ & $\mathrm{~cm} \mathrm{~s}^{-} 2$ \\
\hline mass & 23 & Ha98 & $15-20$ & $\mathrm{Mo} 00$ & $M_{\odot}$ \\
\hline$T_{\star}(\tau \sim 20)$ & 32900 & $\mathrm{Mo} 00$ & & & $\mathrm{~K}$ \\
\hline$T_{\text {eff }}(\tau \sim 2 / 3)$ & 26000 & Cr95 & $28500 \pm 2000$ & Cr97 & K \\
\hline$R_{\star}$ & 20.6 & Mo00 & & & $R_{\odot}$ \\
\hline$R_{\text {eff }}$ & 27 & Cr95 & & & $R_{\odot}$ \\
\hline$T_{\mathrm{e}}$ & 9400 & Ch92 & 10000 & $\mathrm{a}$ & $\mathrm{K}$ \\
\hline$v_{\infty}$ & 950 & $\mathrm{Mo00}$ & 800 & $\mathrm{a}$ & $\mathrm{km} \mathrm{s}^{-1}$ \\
\hline$Z$ & 1.005 & Mo00 & 1.0 & $\mathrm{a}$ & $\mathrm{e}$ \\
\hline$\gamma$ & 1.003 & $\mathrm{Mo00}$ & 1.0 & $\mathrm{a}$ & \\
\hline$\mu$ & 3.18 & $\mathrm{Mo00}$ & 1.5 & $\mathrm{a}$ & $m_{\mathrm{e}}$ \\
\hline$\dot{M}$ & $2.410^{-5}$ & $\mathrm{Mo00}$ & $410^{-7}$ & $\mathrm{a}$ & $M_{\odot} \mathrm{yr}^{-1}$ \\
\hline$n_{\mathrm{e}}$ at the shock front & 7665 & $\mathrm{~d}$ & 23236 & $\mathrm{~d}$ & $\mathrm{~cm}^{-3}$ \\
\hline$g_{\mathrm{ff}}(5 \mathrm{GHz})$ & 5.018 & $\mathrm{~d}$ & 5.072 & $\mathrm{~d}$ & \\
\hline$g_{\mathrm{ff}}(1.4 \mathrm{GHz})$ & 5.720 & $\mathrm{~d}$ & 5.774 & d & \\
\hline$g_{\mathrm{ff}}(350 \mathrm{MHz})$ & 6.552 & $\mathrm{~d}$ & 6.576 & $\mathrm{~d}$ & \\
\hline$R_{5} \mathrm{GHz}$ & 45 & $\mathrm{~d}$ & 5 & $\mathrm{~d}$ & $\mathrm{AU}$ \\
\hline$R_{1.4 \mathrm{GHz}}$ & 110 & $\mathrm{~d}$ & 10 & $\mathrm{~d}$ & $\mathrm{AU}$ \\
\hline$R_{350 \mathrm{MHz}}$ & 286 & $\mathrm{~d}$ & 33 & d & $\mathrm{AU}$ \\
\hline
\end{tabular}

Notes:

d: derived; a: assumed; Ch92: Churchwell et al. 1992; Cr95: Crowther et al. 1995; Cr97: Crowther 1997; Ha98: Hamann \& Koesterke 1998; Mo00: Morris et al. 2000; Ni98: Niemela et al. 1998; Po87: Pollock 1987 (corrected for distance); Po95: Pollock et al. 1995; Se00: this work; Sm96: Smith et al. 1996; Wi97: Williams et al. 1997.

as listed in Table 4. In Fig. 5 we present a pole-on sketch of the system. For a distance of $650 \mathrm{pc}$ to the system, the $350-5000-\mathrm{MHz}$ radiophotospheres of both components of the binary are smaller than their separations from the non-thermal source between them.

As mentioned above, both the stellar wind model of Wright \& Barlow (1975) and the colliding winds model of Usov (1992) are for smooth winds. A clumpy wind will produce asymmetries in the radiophotospheres. Furthermore, in the frame of Usov's equal ram-pressure colliding-wind model, if a clumpy WN8 wind collides with a smooth OB wind, the clumps will penetrate further into the $\mathrm{OB}$ wind than a smooth WN8 wind would do, so that the interaction region will be closer to the OB star. The result is a more extended interaction region.

As the cartoon shows, the $350-\mathrm{MHz}$ flux density could be significantly affected by free-free absorption in the WN8 system, if our line of sight to the interaction region 
Table 5. Flux densities and sizes of WR 147, used to plot the spectral energy distribution (Fig. 4). Note that the values listed here are from observations at different epochs, and therefore, may be affected by the variability of the WR 147 system

\begin{tabular}{|c|c|c|c|c|c|c|}
\hline & $N+S$ & ref. & $S$ & ref. & $N$ & ref. \\
\hline$S_{0.45 \mathrm{~mm}}(\mathrm{mJy})$ & $1,200 \pm 200$ & Wi97 & & & & \\
\hline$S_{0.8 \mathrm{~mm}}(\mathrm{mJy})$ & $357 \pm 50$ & Wi97 & & & & \\
\hline$S_{1.1 \mathrm{~mm}}(\mathrm{mJy})$ & $292 \pm 20$ & Wi97 & & & & \\
\hline$S_{1.3 \mathrm{~mm}}(\mathrm{mJy})$ & $280 \pm 30$ & Wi97 & & & & \\
\hline$S_{2 \mathrm{~mm}}(\mathrm{mJy})$ & $275 \pm 40$ & Wi97 & & & & \\
\hline$S_{7 \mathrm{~mm}}(\mathrm{mJy})$ & $82.8 \pm 1.1$ & Co96 & & & & \\
\hline$S_{1.3 \mathrm{~cm}}(\mathrm{mJy})$ & 57.3 & Sk99 & 53.9 & Sk99 & 3.4 & Sk99 \\
\hline$S_{2 \mathrm{~cm}}(\mathrm{mJy})$ & 49.6 & Sk99 & 41.8 & Sk99 & 7.8 & Sk99 \\
\hline$S_{3.6 \mathrm{~cm}}(\mathrm{mJy})$ & 41.2 & Co99 & $31.3 \pm 0.5$ & Co99 & $9.9 \pm 0.4$ & Co99 \\
\hline$S_{6 \mathrm{~cm}}(\mathrm{mJy})$ & $35.4 \pm 0.4$ & $\mathrm{Se} 00$ & $21.4 \pm 0.4$ & Ch92 & $11.9 \pm 0.4$ & Ch92 \\
\hline$S_{18 \mathrm{~cm}}(\mathrm{mJy})$ & $16.3 \pm 0.2$ & Wi97 & & & $11.4 \pm 0.6$ & Wi97 \\
\hline$S_{21 \mathrm{~cm}}(\mathrm{mJy})$ & $26.4 \pm 0.3$ & $\mathrm{Se} 00$ & & & & \\
\hline$S_{92 \mathrm{~cm}}(\mathrm{mJy})$ & $16 \pm 4$ & $\mathrm{Se} 00 \mathrm{~b}$ & & & & \\
\hline size at $3.6 \mathrm{~cm}$ (mas) & & & $230 \times 200$ & Co99 & $410 \times 200$ & Co99 \\
\hline size at $6 \mathrm{~cm}$ (mas) & & & $170(\mathrm{E}-\mathrm{W}) \times 253$ & Wi97 & $267(\mathrm{E}-\mathrm{W}) \times 79$ & Wi97 \\
\hline
\end{tabular}

\section{References:}

Ch92: Churchwell et al. 1992; Co96: Contreras et al. 1996; Co99: Contreras et al. 1999; Sk99: Skinner et al. 1999; Se00: this work; Se00b: Paper II; Wi97: Williams et al. 1997.

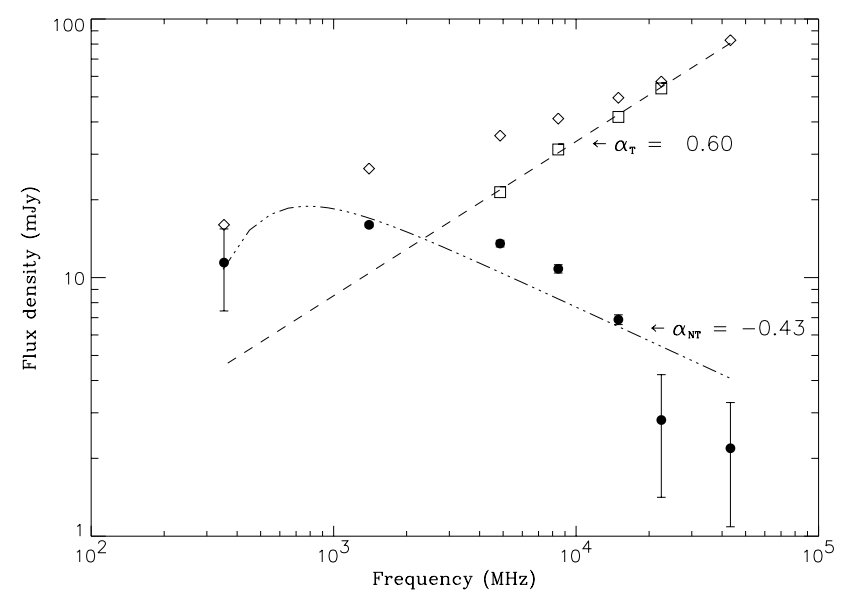

Fig. 4. The spectral energy distribution of WR 147. The dashed line is a fit to the thermal source data $(\square), \alpha_{\text {thermal }}=$ 0.6. The observed total flux densities, $S_{\text {total }}$, are marked with $\diamond$, while $\bullet$ represents the implied non-thermal flux densities, $S_{\mathrm{NT}}=S_{\text {total }}-S_{\text {fit }}$. The $(-\cdots-)$ line is a fit to the non-thermal flux densities of the synchrotron emission, with $\alpha_{\mathrm{sync}}=-0.43$, affected by free-free absorption at frequencies below $1 \mathrm{GHz}$

is passing through the $\mathrm{WN} 8350-\mathrm{MHz}$ radiophotosphere. Free-free absorption of the shock front radiation by the OB radiophotosphere depends strongly on the lateral extent of the shock front.

\subsection{Origin of the non-thermal emission in WR 147}

The flux density of synchrotron emission from particles with a power-law energy distribution, $N(E) d E=$ $K E^{\gamma} d E$, also has a power law form of $S_{\nu} \propto \nu^{\alpha}$, where $\alpha=-(\gamma-1) / 2$. However, Fig. 4 shows lower fluxes both at low and high frequencies. The high frequency turn-over, around $22 \mathrm{GHz}$ is caused by the energy cut-off in the electron distribution, i.e., there are not enough relativistic electrons to generate significant synchrotron emission at higher frequencies. The low-frequency turn-over can be due to several causes.

The source can be optically thick at low frequency and become self-absorbed. The critical frequency where this mechanism occurs depends on the flux density, $S_{\nu}$, in mJy, the size of the source, $\theta$, in arcsec, and the strength of the magnetic field, $B$, in Gauss, according to (Williams 1963):

$\nu_{\mathrm{sa}} \simeq 2.145\left(\frac{S_{\nu_{\mathrm{sa}}}}{\theta^{2}}\right)^{2 / 5} B^{1 / 5} \mathrm{MHz}$.

If we assume that $\nu_{\mathrm{sa}} \approx 1400 \mathrm{MHz}, B=5 \mathrm{mG}$, and $S_{\nu_{\mathrm{sa}}} \approx 17 \mathrm{mJy}$, then the minimum observable angular size of the non-thermal source in WR 147, if it is undergoing synchrotron self-absorption, is about 0.3 mas. The 1.6-GHz MERLIN map of Williams et al. (1997), shows a three orders of magnitude larger source. Therefore, because the source is not compact enough, we can reject the synchrotron self-absorption mechanism to be responsible for the low energy turn-over in WR 147.

When the thermal plasma has a sufficiently high density, the speed of the emitting particles cannot approach the phase velocity (i.e., the wave crest that it emits) closely. This will suppress the synchrotron emission. The frequency at which this Razin-Tsytovich effect occurs is (Pacholczyk 1970):

$\nu_{R} \simeq 20 \frac{n_{\mathrm{e}}}{B} \mathrm{~Hz}$

where $n_{\mathrm{e}}$ is the electron density in $\mathrm{cm}^{-3}$ :

$n_{e}=\frac{\dot{M}}{4 \pi r^{2} v_{\infty} \mu m_{H}} \mathrm{~cm}^{-3}$,

and $B$ is magnetic field in Gauss. Applying the values for the components of WR 147 to Eq. (8), the minimum 


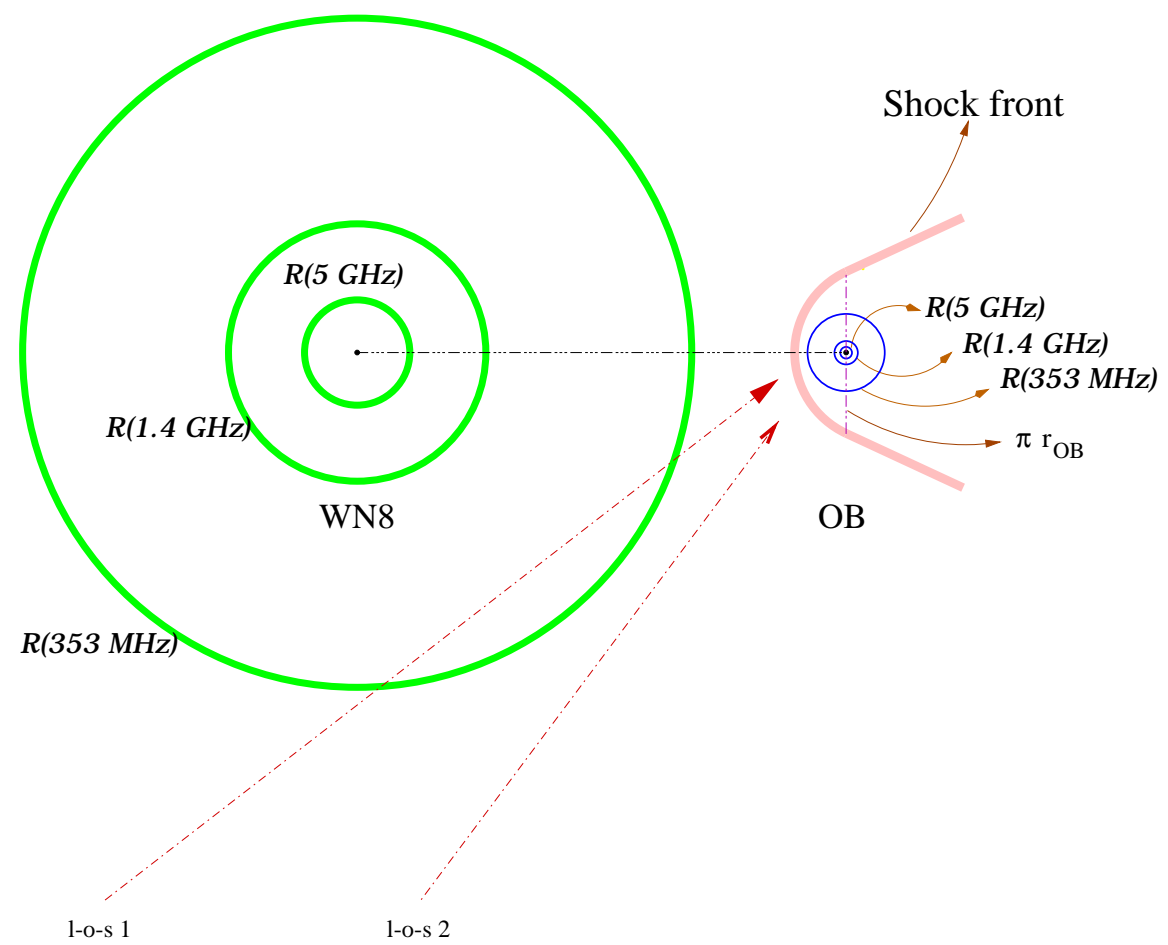

Fig. 5. Pole-on view of the geometry of the WR 147 system. Shown are radiophotospheres and the shock front due to smooth winds. Clumpiness in the wind will produce asymmetries of the radiophotospheres and a more extended interaction region. Depending on our line of sight to the system, the radiophotosphere can cause free-free absorption of the radiation from the shock front (l-o-s 1) or not (l-o-s 2)

magnetic field required to be able to observe the 1.4-GHz radiation, is $0.3 \mathrm{mG}$. However, from the equipartition of energy, the magnetic field at the collision region is $B_{\min } \approx$ $5 \mathrm{mG}$, which is more than 10 times larger than needed to overcome the Razin-Tsytovich effect. Therefore, we can also reject the Razin-Tsytovich effect as a cause of the suppression of the synchrotron emission at low frequency.

Another mechanism to explain this low-frequency turn-over is free-free absorption. Synchrotron emission absorbed by a screen of matter between the source and the observer will have the shape (Paper I; Skinner et al. 1999):

$S_{\nu}=C \nu^{\alpha} e^{\left(-\tau_{0} \nu^{-2.1}\right)}$.

Fitting this function to the non-thermal flux density, shown in Fig. 4, gives a poor fit with reduced $\chi^{2} \approx 8$, and a spectral index $\alpha=-0.43$.

Skinner et al. (1999) suggested that their nearsimultaneous radio data were best fitted with absorbed synchrotron emission from a nearly mono-energetic population of electrons. If this is the case, then WR 147 is among the very rare objects producing mono-energetic synchrotron emission. A more common mechanism may still be involved in the WR 147 system. De Bruyn (1976) showed that if the relativistic electrons in a synchrotron emitting zone are mixed with thermal ones, different spectral shapes can be achieved. He assumed spherical symmetry and isotropic relativistic particles. The magneticfield strength $B$, the relativistic electron density coefficient $K$, and the thermal electron density $n_{\mathrm{e}}$ in the source all vary with the distance from the source, $r$, according to
$B=B_{0}\left(r / r_{0}\right)^{-m}, K=K_{0}\left(r / r_{0}\right)^{-n}$ and $n_{\mathrm{e}}=n_{0}\left(r / r_{0}\right)^{-p}$. The synchrotron spectrum will then be of the form (de Bruyn 1976):

$S_{\nu} \propto \nu^{(13-4 n-2 n-2 m-2 p-\gamma-2 \gamma(m+n)) /(2-4 p)}$

with the limits:

$2-n-m(\gamma+1) / 2 \gtrless 0$ for $1-2 p \gtrless 0$.

By varying $\gamma, m, n, p$, a similar shape of spectral distribution as in Fig. 4 could be reproduced. The exact values of these parameters will need a separate study. As clumpiness in the wind will increase the likelihood of a mixing of the particles, this model deserves further investigation.

Attenuation by the $350-\mathrm{MHz}$ radiophotosphere can also add to the absorption. This can happen if our line of sight to the system has an inclination lower than the angular size of the WN8 or OB stars at $350 \mathrm{MHz}$ as seen from the shock front, i.e., $\sim 40^{\circ}$. We conclude that free-free absorption is the most likely cause for the low frequency fall-off in the radio spectrum.

\subsection{Variability of the 1.4 and $5-G H z$ flux densities}

\subsubsection{A periodic variation?}

We submitted both the 1.4 and 5-GHz data to a Scargle periodogram using STARLINK's PERIOD program and find that there is a suggestion of a periodicity of $7.9 \mathrm{yr}$ in the 1.4-GHz data (Fig. 6). The resulting sine-wave fit is shown in Fig. 7. We note that the fit has a rather large 


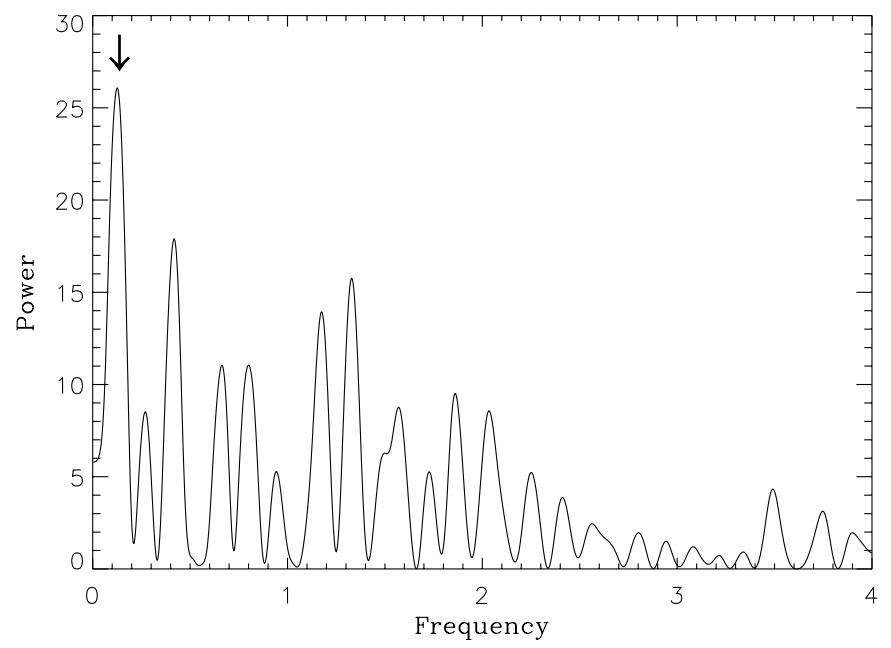

Fig. 6. The power spectrum resulting from the Scargle periodogram for the 1.4- $\mathrm{GHz}$ data of WR 147. The arrow points to the frequency which corresponds to a period of $7.9 \mathrm{yr}$

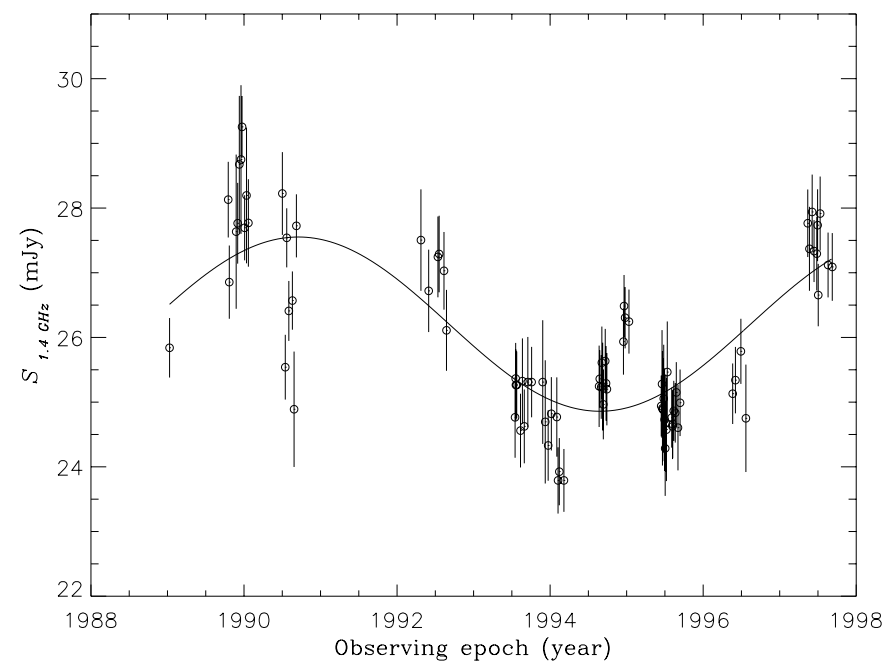

Fig. 7. A sinusoid with a period of $7.9 \mathrm{yr}$ and a semi-amplitude of $1.35 \pm 0.08$ mJy fitted to the $1.4-\mathrm{GHz}$ light curve of WR 147 . The reduced $\chi^{2}$ of the fit is 1.92

reduced $\chi^{2}$ value of 1.92 and that the data cover only one period.

As mentioned earlier, the observed WSRT flux density of WR 147 is a composite of the thermal and non-thermal components. From the spectral indices and flux densities at other frequency (Skinner et al. 1999; Williams et al. 1997, etc.), we derive that at $1.4 \mathrm{GHz}$ the flux density of the thermal component in WR 147 is roughly half that of the non-thermal component. As a result, if the amplitude of the WSRT flux-density variation is lower than the flux density of the thermal source, it will be difficult to distinguish the varying source. This is unlike the situation in WR 146 (Paper I), where we can readily pin-point the non-thermal component to be the varying source, as at $1.4 \mathrm{GHz}$ the flux-density variation has an amplitude which is more than 20 times larger than the thermal flux density.
The 2.7-mJy peak-to-peak variation of the 1.4-GHz sinusoid corresponds to $\sim 10 \%$ of the total $1.4-\mathrm{GHz}$ flux density of $26.4 \mathrm{mJy}$. If we adopt the fitted value of the thermal source, we obtain $S_{\text {thermal }}=9.4 \mathrm{mJy}$ and $S_{\mathrm{NT}}=17 \mathrm{mJy}$. The peak-to-peak variation then corresponds to $\sim 27 \%$ of the thermal source, or $\sim 15 \%$ of the non-thermal source. Contreras \& Rodríguez (1999) observed a $25 \%$ increase in the 8.4-GHz thermal flux densities in two epochs of 1995 July and 1996 December. Could it be that the 1.4-GHz sinusoidal variation is related to their thermal flux-density increase, as they have similar magnitudes?

In the case that the $1.4-\mathrm{GHz}$ sinusoidal variation is due to the thermal source, the positive spectral index means that we should see a similar sinusoidal variation at $5 \mathrm{GHz}$ but with larger amplitude, because $S_{\nu} \propto \nu^{\alpha}$. Taking a spectral index $\alpha=0.6$, we obtain a $5-\mathrm{GHz}$ semi-amplitude of $2.88 \mathrm{mJy}$. However, should the variability be due to the non-thermal source, then, if we adopt a spectral index $\alpha=-0.43$, the expected $5-\mathrm{GHz}$ semi-amplitude is 0.79 mJy. As can be seen in Fig. 8, the observed $5-\mathrm{GHz}$ variations are clearly inconsistent with a sinusoidal variation of thermal origin. However the variations are also not consistent with a non-thermal origin. The observed 5 and $1.4-\mathrm{GHz}$ variations may therefore be dominated by a stochastic process with shorter characteristic timescale. There would then be no correlation expected between the 5 and 1.4-GHz flux densities, because they were not observed simultaneously. This possibility will be discussed further below. To confirm the sinusoid, we need to extend our present data set with more and regular monitoring of the system across the radio frequency range for at least another ten years.

\subsubsection{Stochastic variations}

The shorter-term scatter of the data may reflect irregular, stochastic variations caused by inhomogeneities in the winds of the binary components entering the windcollision region. We observe a typical timescale for the flux-density variation of 60 days. We consider several mechanisms that can be responsible for the increase and decrease of the flux density.

$i$. Variability in synchrotron emission. The flux-density increase can be a result of increased emission in the synchrotron process. If the time needed to accelerate the particles is $t_{\text {acc }} \ll 60 \mathrm{~d}$, then the timescale of 60 days might reflect the size of the clumps. For a wind velocity of $950 \mathrm{~km} \mathrm{~s}^{-1}, 60$ days corresponds to clump size of about $30 \mathrm{AU}$, or about 55 mas. A clump of this size corresponds approximately to a $5-10 \%$ increase in the volume of the collision zone. A smaller clump size would yield a smaller timescale, but the corresponding variability would also be less pronounced, as the effect will be diluted.

Relativistic electrons moving in a magnetic field strength of $5 \mathrm{mG}$ and radiating at a synchrotron characteristic frequency of $1.4 \mathrm{GHz}$ have an energy of about 


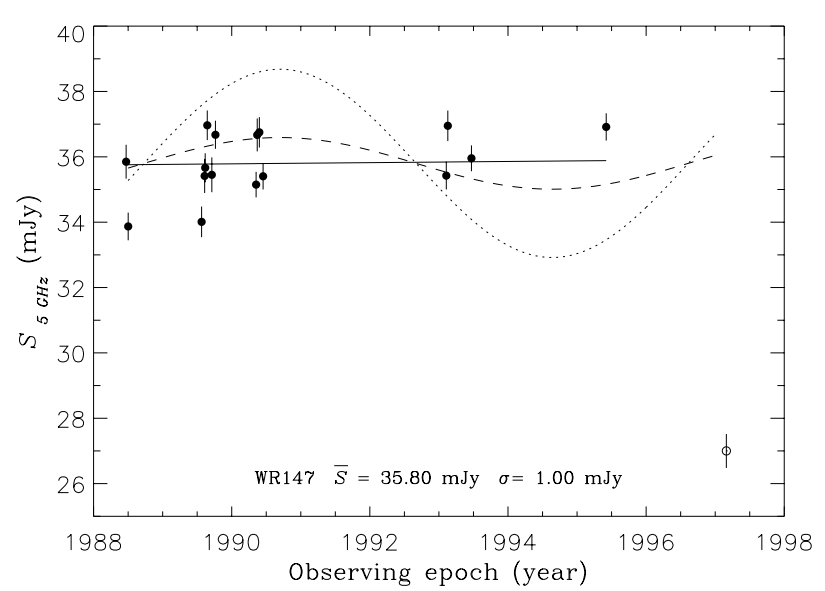

Fig. 8. 5-GHz light curve of WR 147 . The 1997 data point (o) is not used in the analysis. The sinusoids are discussed in Sect. 4.3 and represent the expected variation in the $5-\mathrm{GHz}$ light curve for two different radiation sources, a thermal source (.....) and a non-thermal source (-- ). Since the spectral index of a thermal steady-wind source is about $\alpha=0.6$ (where $S_{\nu} \propto$ $\nu^{\alpha}$ ), flux-density variability at lower frequency will be observed with larger amplitude than at higher frequency. For a nonthermal source, with a spectral index much smaller than 0.6 or even negative, the amplitude of the variability will be relatively smaller at higher frequency. The period value is taken from the analysis of the 1.4-GHz data (see Sect. 4.3)

$210^{-3}$ erg. For these electrons, the timescale of the synchrotron losses is, following Pacholczyk (1970):

$t_{\mathrm{S}}=\frac{1}{2.3710^{-3} E B^{2}} \sec$

i.e., $2.510^{3} \mathrm{yr}$.

The timescale of Inverse-Compton (IC) losses, however, is (Pacholczyk 1970):

$t_{\mathrm{IC}}=\frac{4 \pi r^{2} c}{3.9710^{-2} L_{\star} E}$.

Using the parameter values for the WR and OB stars listed in Table 4 we obtain $t_{\mathrm{IC}}(\mathrm{WR}) \approx 26 \mathrm{yr}$ and $t_{\mathrm{IC}}(\mathrm{OB}) \approx 4.5 \mathrm{yr}$. These timescales are still much longer than the shortest variability timescale in the data. Therefore, we conclude that both synchrotron losses and IC losses are not responsible for the flux-density decrease in WR 147.

ii. Variability in the free-free absorption. Changes in the optical depth in the line of sight will cause variations in free-free absorption of the radiation from the collidingwinds region, and, therefore, can be responsible for both increase and decrease in the flux density. This can be due to, e.g., high-density clumps in the stellar wind between the observer and the colliding-wind region, giving "eclipse" effects. The timescale for this will vary depending on the transverse size and velocity of the clump (cf. Fig. 5). As an illustration, a homogenous clump of 55 mas (=30 AU), moving with the WR wind velocity, at an angle of $70^{\circ}$ to the line of sight, will cause a drop in the flux-density for $\sim 58$ days, before the flux density goes up again. Variability in the free-free absorption will obviously be more pronounced at low frequencies.

iii. Plasma outflow. The relativistic particles produced in the colliding-wind region will eventually flow out with the plasma along the shock-front cone (cf. Fig. 5). This will cause a flux-density decrease, as the magnetic field is weaker further away from the stars. The flow time in the colliding-wind region is (Eichler \& Usov 1993):

$$
\begin{aligned}
t_{\mathrm{f}} \simeq \frac{r_{\mathrm{OB}}}{v_{\infty}(\mathrm{WR})} \simeq & 0.510^{5} \eta^{1 / 2}\left(\frac{D_{\mathrm{WR}-\mathrm{OB}}}{10^{13} \mathrm{~cm}}\right) \\
& \times\left(\frac{v_{\infty}(\mathrm{WR})}{210^{8} \mathrm{~cm} \mathrm{~s}^{-1}}\right)^{-1} \mathrm{sec},
\end{aligned}
$$

i.e., $\sim 80$ days.

We conclude that the flux-density increase is most likely due to inhomogeneities in the wind causing increased emission and variable free-free absorption in the line of sight. The flux-density decrease could be due to both inhomogeneities in the wind, causing variable freefree absorption and plasma outflow.

\section{Summary and future work}

We summarize our results as follows:

$i$. We confirm previous studies that the non-thermal source in WR 147 is synchrotron emission, arising from relativistic particles accelerated by shocks in the region where the winds of the binary components collide. A similar mechanism, modelled by Eichler \& Usov (1993), has been suggested by various authors to take place in the binary systems WR 140 and WR 146. We suggest that the relativistic particles are mixed with thermal particles which cause free-free absorption of the synchrotron radiation; ii. There are irregular, stochastic variations caused by inhomogeneities in the winds, i.e., clumps entering the colliding-wind region or the line of sight, and outflow of plasma from the colliding-wind region; and

iii. There is a possibility of a sinusoidal variation of about 8-yr period.

To improve the understanding of the system, and to test the conclusions above, we offer as suggestion for future work: monitoring the system on a monthly basis for at least ten more years, simultaneously across the radio frequency range, but especially at frequencies below $1 \mathrm{GHz}$, to investigate the reality of the sinusoidal pattern observed at $1.4 \mathrm{GHz}$ and to get better spectral energy distributions of the non-thermal and thermal radio components.

Acknowledgements. Financial support for DYASG from the Rotary Foundation, the University of Utrecht, and the Leids Kerkhoven-Bosscha Fonds is gratefully acknowledged. We thank the referee, Dr. Joe Cassinelli, for very constructive comments, and Dr. Nick Whyborn for critical reading of the manuscript. This research has made use of the SIMBAD database, operated at the CDS, Strasbourg, France. The WSRT is operated by the Netherlands Foundation for Research in Astronomy (NFRA) which is financially supported by the Netherlands Organization for Scientific Research (NWO). 


\section{References}

Baars, J. W. M., Genzel, R., Pauliny-Toth, I. I. K., \& Witzel, A. 1977, A\&A, 61,99

de Bruyn, A. G. 1976, A\&A, 52, 439

Churchwell, E. B., Bieging, J. H., van der Hucht, K. A., et al. 1992, ApJ, 393, 329

Contreras, M. E., \& Rodríguez, L. F. 1999, ApJ, 515, 762

Contreras, M. E., Rodríguez, L. F., Gómez, Y., \& Velaśquez, A. 1996, ApJ, 469, 329

Crowther, P. A. 1997, The Effective Temperatures of Hot Stars, in Proc. IAU Symp. 189, Fundamental Stellar Properties: The Interaction Between Observation and Theory, ed. T. R. Bedding, A. J. Booth, \& J. Davis (Kluwer, Dordrecht), 137

Crowther, P. A., Smith, L. J., Hillier, D. J., \& Schmutz, W. 1995, A\&A, 293, 403

Eichler, D., \& Usov, V. V. 1993, ApJ, 402, 271

Hamann, W.-R., \& Koesterke, L. 1998, A\&A, 333, 251

van der Hucht, K. A., Williams, P. M., Spoelstra, T. A. Th., \& Swaanenvelt, J. P. 1995, Radio monitoring of the nonthermal Wolf-Rayet objects WR 125, WR 146 and WR 147, in Proc. IAU Symp. 163, Wolf-Rayet Stars: Binaries, Colliding Winds, Evolution, ed. K. A. van der Hucht, \& P. M. Williams (Kluwer, Dordrecht), 559

van der Hucht, K. A., Schrijver, H., Stenholm, B., et al. 1997, New Astron., 2, 245

Ignace, R., Cassinelli, J. P., \& Bjorkman, J. E. 1998, ApJ, 505,910

Jardine, M. M., Allen, H. R., \& Pollock, A. M. T. 1996, A\&A, 314,594

Leitherer, C., \& Robert, C. 1991, ApJ, 377, 629

Maheswaran, M., \& Cassinelli, J. P. 1994, ApJ, 421, 718

Miley, G. K. 1991, Ann. Rev. Astron. Astroph., 18, 165

Moran, J. P., Davis, R. J., Bode, M. F., et al. 1989, Nature, 340,449

Morris, P. W., van der Hucht, K. A., Crowther, P. A., et al. 2000, A\&A, 353, 624

Niemela, V. S., Shara, M. M., Wallace, D. J., Zurek, D. R., \& Moffat, A. F. J. 1998, AJ, 115, 2047

Noordam, J. E. 1994, NEWSTAR Cookbook

Pacholczyk, A. G. 1970, Radio Astrophysics (Freeman, San Francisco)
Pollock, A. M. T. 1987, ApJ, 320, 283

Pollock, A. M. T., Haberl, F., \& Corcoran, M. F. 1995, The Rosat PSPC survey of the Wolf-Rayet stars, in Proc. IAU Symp. 163, Wolf-Rayet Stars: Binaries, Colliding Winds, Evolution, ed. K. A. van der Hucht, \& P. M. Williams (Dordrecht: Kluwer), 512

Setia Gunawan, D. Y. A., van der Hucht, K. A., Stickland, D. J., et al. 1995a, IUE monitoring of WR 140 (WC6+O4-5), in Proc. IAU Symp. 163, Wolf-Rayet Stars: Binaries, Colliding Winds, Evolution, ed. K. A. van der Hucht, \& P. M. Williams (Kluwer, Dordrecht), 466 Setia Gunawan, D. Y. A., van der Hucht, K. A., Stickland, D. J., et al. 1995b, An ultraviolet radial velocity orbit for WR 140 (WC6+O4-5), in Proc. IAU Symp. 163, Wolf-Rayet Stars: Binaries, Colliding Winds, Evolution, ed. K. A. van der Hucht, \& P. M. Williams (Kluwer, Dordrecht), 508

Setia Gunawan, D. Y. A., de Bruyn, A. G., van der Hucht, K. A., \& Williams, P. M. 2000, A\&A, 356, 676 (Paper I)

Skinner, S. L., Itoh, M., Nagase, F., \& Zhekov, S. A. 1999, ApJ, 524, 394

Smith, L. F., Shara, M. M., \& Moffat, A. F. J. 1996, MNRAS, 281,163

Taylor, A. R., Goss, W. M., Coleman, P. H., van Leeuwen, J., \& Wallace, B. J. 1996, ApJS, 107, 239

Usov, V. V. 1992, ApJ, 389, 635

Wendker, H. J., Higgs, L. A., \& Landecker, T. J. 1991, A\&A, 241,551

White, R. L., \& Becker, R. H. 1995, ApJ, 451, 352

Williams, P. J. S. 1963, Nature, 200, 56

Williams, P. M. 1996, Radio emission from Wolf-Rayet and OB stellar winds, in Radio Emission from the Stars and the Sun, ed. A. R. Taylor, \& J. M. Paredes, PASPC, 93, 15

Williams, P. M., van der Hucht, K. A., Pollock, A. M. T., et al. 1990, MNRAS, 243, 662

Williams, P. M., van der Hucht, K. A., \& Spoelstra, T. A. Th. 1994, A\&A, 291, 805

Williams, P. M., Dougherty, S. M., Davis, R. J., et al. 1997, MNRAS, 289, 10

Wright, A. E., \& Barlow, M. J. 1975, MNRAS, 170, 41

Zoonematkermani, S., Hefland, D. J., Becker, R. H., White, R. L., \& Perley, R. A. 1990, ApJS, 74, 181 